\title{
Effects of selenium supplementation on paraoxonase-1 and myeloperoxidase activity in subjects with cardiovascular disease: the Selenegene study, a double-blind randomized controlled trial
}

\author{
Amirhossein Mirmohammadsadeghi ${ }^{1}$, Mojgan Gharipour ${ }^{2}$, Hamidreza Roohafza ${ }^{3}$, \\ Minoo Dianatkhah ${ }^{4}$, Masoumeh Sadeghi ${ }^{5}$
}

\author{
${ }^{1}$ Cardiac Rehabilitation Research Center, medical student, Hormozgan University \\ of Medical Sciences, Hormozgan, Iran \\ ${ }^{2}$ Isfahan Cardiovascular Research Center, Cardiovascular Research Institute, Isfahan \\ University of Medicine Sciences, Isfahan, Iran \\ ${ }^{3}$ Psychosomatic Research Center, Isfahan University of Medicine Sciences, Isfahan, Iran \\ ${ }^{4}$ Heart Failure Research Center, Cardiovascular Research Institute, Isfahan University \\ of Medicine Sciences, Isfahan, Iran \\ ${ }^{5}$ Cardiac Rehabilitation Research Center, Cardiovascular Research Institute, \\ IsfahanUniversity of Medicine Sciences, Isfahan, Iran
}

Submitted: 18 January 2018

Accepted: 19 July 2018

Arch Med Sci Atheroscler Dis 2018; 3: e112-e118

DOI: https://doi.org/10.5114/amsad.2018.77820

Copyright (c) 2018 Termedia \& Banach

\begin{abstract}
Introduction: We previously highlighted the potential link between supplementation with selenium, as an antioxidant trace element, and changes in the levels of paraoxonase (POX1) and myeloperoxidase (MPO), as an antioxidant enzyme, in patients with documented cardiovascular disease (CVD). The aim of this study was to determine the effects of selenium supplementation on POX1 and MPO activity in patients with cardiovascular diseases (CVDs).

Material and methods: A total of 160 eligible patients were enrolled in the study. After performing some laboratory tests, including the measurement of blood selenium, triglyceride, cholesterol, and low- and high-density lipoprotein levels, the patients received $200 \mathrm{mg}$ tablets of either selenium yeast or placebo. The medicines were taken orally, once daily after a meal for 60 days. Four weeks after the initial visit, the patients were invited for a follow-up visit, and interviews and non-laboratory evaluations, similar to those performed at baseline, were repeated. Compliance of patients for using selenium and placebo was measured by telephone. Medication compliance rates were monitored by telephone. The final assessments were conducted eight weeks after the beginning of the study.

Results: There was no significant difference in cholesterol levels between in tervention and control groups $(p=0.87)$. No significant changes in selenium levels were observed in either the selenium or the placebo group after the intervention ( $p=0.44$ and $p=0.48$, respectively). The two groups had a significant difference in terms of POX1 level $(p=0.039)$. No such difference was present in the case of MPO levels. Moreover, comparison of the values before and after the intervention showed no significant differences in the mean levels of any of the measured parameters.

Conclusions: According to the obtained results, the increased POX1 levels after selenium supplementation could be attributed to the positive effect of selenium on inhibiting lipid peroxidation as part of the complicated pathophysiology of CVD.
\end{abstract}

Corresponding author: Prof. Masoumeh Sadeghi Cardiac Rehabilitation Research Center Cardiovascular Research Institute Isfahan University of Medicine Sciences Isfahan, Iran E-mail: sadeghimasoumeh@ gmail.com

Key words: selenium, cardiovascular disease, myeloperoxidase, paraoxonase-1. 


\section{Introduction}

Cardiovascular disease (CVD) is considered as the second cause of morbidity and mortality in the developed and developing countries [1, 2]. Previous studies revealed the main role of oxidative stress in the incidence of CVD. So, it seems that antioxidants could take part in prevention of CVD. Selenium is a rare trace element whose deficiency may exert adverse health effects. However, there is still controversy over the exact effects of selenium deficiency on health, particularly in relation to cardiovascular diseases (CVDs) [3]. Despite the significant role of selenium in preventing certain diseases, a previous study indicated that the average level of selenium in soil in Iran (0.23 ppm) is below the worldwide average (0.40 ppm) [4].

Paraoxonase (POX1) has been found to possess some anti-inflammatory, anti-oxidative, anti-atherogenic, anti-diabetic, anti-microbial, and organophosphate-hydrolyzing properties [5]. Moreover, POX1 is known to function as both a lactonase and an arylesterase $[6,7]$.

Myeloperoxidase (MPO) is another key leukocyte-derived enzyme that catalyzes the formation of a number of reactive oxygen species [8]. MPO-catalyzed reactions have been attributed to potentially proatherogenic biological activities throughout the evolution of CVDs, i.e. through the beginning, circulation, and acute complication phases of the atherosclerotic process [9]. As a result, MPO and its downstream inflammatory pathways serve as special targets for both prognostication and therapeutic interventions in the prophylaxis of atherosclerotic CVD [10, 11]. Recently, a potential link has been established between supplementation with selenium, as an antioxidant trace element, and changes in the levels of POX1 and MPO, as antioxidant enzymes in patients with documented CVD. The aim of this study was to determine the effects of selenium supplementation on POX1 and MPO activity in patients with CVD.

\section{Material and methods}

\section{Study design and settings}

This was a single-center, double-blind, placebo-controlled, randomized clinical trial. All participants had coronary artery disease according to angiographic findings. In total, 160 eligible patients were consecutively enrolled in this study. The subjects were referred to our research institute from referral heart hospitals in Isfahan, Iran. The trial was approved by the Isfahan University of Medical Sciences and Research Ethics Committee and conforms to the standards currently applied by the Iranian Registry of Clinical Trials (ID: IRCT10252) [12].

\section{Inclusion criteria}

Individuals aged between 30 and 65 years who had lived in Isfahan province for more than 5 years were recruited if angiographic findings confirmed stenosis of over $75 \%$ in one, two, or three vessels.

\section{Exclusion criteria}

The patients were excluded if they were under medical prescription of vitamins or supplements.

\section{Data collection}

The initial interviews and tests included a questionnaire to collect demographic data, medical history, physical activity, smoking habits, anthropometric measurements, and detailed information for a nutritional profile, including dietary selenium intake and biochemical laboratory measurements. Information on food consumption was assessed with a food frequency questionnaire (FFQ). A short, non-quantitative FFQ with 73 items (representing the main food groups) was used to estimate the number of weekly portions of the selected food groups. We did not collect data on portion sizes of food items, as previous studies have shown that data on portion sizes have a negligible contribution to between-person variations in dietary intakes [13].

\section{Intervention}

The patients received either $200 \mathrm{mg}$ selenium yeast tablets or placebo tablets. They were instructed to take the tablets after a meal once daily for 60 days. The placebo tablets had the same color, form, and texture as the selenium tablets. At each visit, the patients in both groups received the number of tablets needed to be taken until the next scheduled appointment. If, for any reason, a treatment interruption occurred, the treatment duration was to be extended until the volunteer had taken 60 tablets. The Nature Company provided selenium yeast in the commercial form. Amin Pharmaceutical Company produced the placebo tablets. All adverse events were recorded [14].

\section{Follow-up}

The participants received follow-up phone calls and briefly asked about the occurrence of any adverse events every 2 weeks. Four weeks after the initial visit, they were invited to visit Isfahan Cardiac Rehabilitation Research Center where interviews and non-laboratory evaluations similar to those performed at baseline were conducted. Moreover, all evaluations, including blood tests, were repeated 8 weeks after the baseline visit. 


\section{Randomization}

A total of 130 individuals were randomly allocated to two groups at a $1: 1$ ratio. The placebo tablets, which looked similar to selenium tablets, were provided by a pharmacist. The tablets were packaged by pharmaceutical companies in the same numerical packages and were recognized with four different codes. Both the executives and interviewers were unaware of the coding. The latter decided which set of participants would form the placebo group and which would be the intervention group by flipping a coin. As the volunteers were recruited by the medical staff, they were assigned a number that sequentially corresponded to a treatment box. In this way, a strategy of numbered boxes was used for sequence concealment.

\section{Blinding}

All patients, interviewers, and staff involved in outcome assessment were blinded to treatment. The blinding was conducted using the described strategy of allocation concealment by numbered boxes. In brief, numbers were assigned to volunteers' treatments, but only one pharmacist, who was not involved in the tasks, was aware of the content of each numbered box.

Compliance of patients for using selenium and placebo was measured by telephone every week and all subjects who stopped using their selenium or placebo were excluded from the study.

Determination of POX1 and MPO activity: POX1 activity was measured with phenyl acetate as the substrate [14]. The assay tube contained $1000 \mu \mathrm{l}$ of $0.1 \mathrm{~mol} / \mathrm{l} \mathrm{Tris}-\mathrm{HCl}$ (pH 8.5), $2 \mathrm{mmol} / \mathrm{l} \mathrm{CaCl}, 2 \mathrm{mmol} / \mathrm{l}$ phenyl acetate, and $3.5 \mu \mathrm{l}$ of serum. The absorbance was continuously monitored at $270 \mathrm{~nm}$ for 2 min. POX1 activity was expressed as millimoles of phenyl acetate hydrolyzed per minute [15].

MPO activity was measured with 3,3',5,5'-tetramethylbenzidine (TMB, Sigma) as the substrate. Briefly, $50 \mu \mathrm{l}$ samples were combined with $240 \mathrm{ml}$ of $0.75 \mathrm{mM} \mathrm{H}_{2} \mathrm{O}_{2}$ (Sigma) and $50 \mathrm{ml}$ of TMB solution (2.9 mM TMB in $14.5 \%$ DMSO and $150 \mathrm{mM}$ sodium phosphate buffer) at $\mathrm{pH}$ 5.4. The absorbance was continuously monitored at $450 \mathrm{~nm}$ for $1 \mathrm{~min}$. MPO activity was expressed as millimoles of TMB oxidized per minute [16].

\section{Outcomes}

The mean serum levels of POX1 and MPO in the target groups were compared before and after the trial.

\section{Stopping rules}

In both groups, all important harmful or unintended effects, such as nausea, vomiting, nail changes, loss of energy, and irritability, were considered as the stopping rules.

\section{Ethics}

The study protocol was explained to all patients and they were recruited only if they provided informed consent. The symptoms and harmful effects of the selenium supplementation were also described to all participants before the end of the study.

\section{Statistical analysis}

All examination results were recorded and stored in digital media for further analysis using Epi Info. Quantitative data were expressed as mean \pm standard deviation (SD). The normal distribution of data was assessed using the Kolmogorov-Smirnov test. Differences between the placebo and control groups were evaluated through t-tests (two tailed). Between-group differences of data departing from normal distribution were analyzed with the Mann-Whitney $U$-test. Categorical variables were presented as frequency counts and compared by a $\chi^{2}$ test. $P$-values less than 0.05 were considered statistically significant. All statistical analyses were performed using SPSS 17.0 (SPSS Inc., Chicago, IL, USA).

\section{Results}

While 160 patients were initially selected, 145 individuals entered the study and data from 130 participants were ultimately analyzed as 15 subjects were lost to follow-up. Table I presents the demographic characteristics and biochemical, blood pressure, and anthropometric measurements in the placebo and selenium groups. There was no significant difference in cholesterol levels between the two groups. The mean triglyceride levels in the placebo and selenium groups were $163.2 \pm 97.8$ and $159.0 \pm 71.8 \mathrm{mg} / \mathrm{dl}$, respectively $(p<0.87)$. The mean low-density lipoprotein cholesterol (LDL-C) and high-density lipoprotein cholesterol (HDL-C) levels were lower in the placebo group than in the Se group $(92.7 \pm 36.0$ vs. 82.6 $\pm 46.2 ; p=0.44$ and $40.4 \pm 8.69$ vs. $42.3 \pm 12.1$; $p=0.56$, respectively). The mean fasting blood sugar (FBS) levels in the placebo and selenium groups were $110.7 \pm 34.1$ and $106.9 \pm 27.1 \mathrm{mg}$ / $\mathrm{dl}$, respectively $(p<0.001)$. The mean body mass index (BMI) was $28.1 \pm 4.18 \mathrm{~kg} / \mathrm{m}^{2}$ in the placebo group and $26.9 \pm 2.27 \mathrm{~kg} / \mathrm{m}^{2}$ in the selenium group $(p=0.22)$. The mean waist-to-hip ratio (WHR) was higher in the placebo group than in the selenium group, but this difference was not significant (101.8 \pm 11.2 vs. $100.1 \pm 6.87 ; p=0.73$ ).

Nutritional habits were assessed and the mean red meat and egg consumption was higher in the 
Table I. Baseline and clinical characteristics and lifestyle habits of participants

\begin{tabular}{|c|c|c|c|}
\hline Variable & $\begin{array}{l}\text { Placebo } \\
(N=21)\end{array}$ & $\begin{array}{l}\text { Selenium } \\
(N=25)\end{array}$ & $P$-value \\
\hline Women, $n(\%)$ & $5(23.8)$ & $3(12.0)$ & 0.29 \\
\hline Age & $57.8 \pm 7.18$ & $55.2 \pm 7.12$ & 0.23 \\
\hline Systolic blood pressure [mm Hg] & $133.6 \pm 17.2$ & $131.9 \pm 18.9$ & 0.75 \\
\hline Diastolic blood pressure [mm Hg] & $80.1 \pm 11.4$ & $82.0 \pm 7.79$ & 0.52 \\
\hline Total cholesterol [mg/dl] & $165.7 \pm 45.1$ & $163.1 \pm 48.9$ & 0.86 \\
\hline Triglyceride [mg/dl] & $163.2 \pm 97.8$ & $159.0 \pm 71.8$ & 0.87 \\
\hline HDL cholesterol [mg/dl] & $40.4 \pm 8.69$ & $42.3 \pm 12.1$ & 0.56 \\
\hline LDL cholesterol [mg/dl] & $92.7 \pm 36.0$ & $82.6 \pm 46.2$ & 0.44 \\
\hline Fasting blood sugar [mg/dl] & $110.7 \pm 34.1$ & $106.9 \pm 27.1$ & 0.68 \\
\hline $\mathrm{BMI}\left[\mathrm{kg} / \mathrm{m}^{2}\right]$ & $28.1 \pm 4.18$ & $26.9 \pm 2.27$ & 0.22 \\
\hline Waist circumference & $101.8 \pm 11.2$ & $100.1 \pm 6.87$ & 0.26 \\
\hline Waist-to-hip ratio & $0.98 \pm 0.08$ & $0.97 \pm 0.05$ & 0.73 \\
\hline \multicolumn{4}{|l|}{ Residency, $n$ (\%): } \\
\hline Urban & $18(85.7)$ & $24(96.0)$ & 0.22 \\
\hline Red meat intake (times/week) & $8.52 \pm 6.26$ & $7.47 \pm 2.87$ & 0.45 \\
\hline Fats & $1.60 \pm 2.45$ & $1.35 \pm 2.32$ & 0.86 \\
\hline Fruit and vegetables & $14.9 \pm 7.22$ & $15.6 \pm 6.71$ & 0.96 \\
\hline Nuts & $3.32 \pm 3.42$ & $3.83 \pm 3.70$ & 0.64 \\
\hline Beans & $1.52 \pm 0.67$ & $2.06 \pm 1.06$ & 0.05 \\
\hline Cereals & $20.6 \pm 8.08$ & $21.9 \pm 6.03$ & 0.51 \\
\hline Dairy products & $13.5 \pm 5.61$ & $14.4 \pm 5.27$ & 0.61 \\
\hline Smoking, $n(\%)$ & $2(9.5)$ & $2(8.0)$ & 0.85 \\
\hline Physical activity, $n$ (\%) & $8(38.1)$ & $5(20.0)$ & 0.30 \\
\hline
\end{tabular}

Values are mean \pm SD unless otherwise stated.

placebo group than in the selenium group $(8.52$ \pm 6.26 vs. $7.47 \pm 2.87 ; p=0.45)$. The mean nut consumption was $3.32 \pm 3.42$ in the placebo group and $15.6 \pm 6.71$ in the selenium group $(p=0.64)$.

Table II displays the selenium, MPO, and POX1 levels in the two groups before and after the study. After the intervention, no significant change in selenium levels was observed in either the Se or the placebo group ( $p=0.44$ and $p=0.48$, respectively). The percentage changes was $-0.07 \pm 0.29$. The intervention and placebo groups had a significant difference in terms of POX1 levels (70.6 \pm 21.9 vs. $84.6 \pm 24.7 ; p=0.039$ ). However, no significant differences in the mean levels of other measured parameters were seen after the intervention.

\section{Discussion}

To the best of our knowledge this double-bind randomized clinical trial for the first time evaluat- ed the role of selenium supplementation in the level of MPO and POX1 in the subjects with metabolic syndrome and a history of CVD. Our main finding is a significant increment in the POX1 levels.

The relations between selenium intake and POX1 and MPO levels are vague and insufficiently discussed. To the best of our knowledge, although several studies have investigated the effect of selenium supplementation on cardiovascular risk factors, this study is the first to examine the effects of selenium supplementation on POX1 and MPO in patients with CVDs. Since POX1 activity decreases in CVDs, hypercholesterolemia, and diabetes, oxidative stress seems to play an essential role in the pathology of these diseases [17].

The results of this study showed a significant difference in POX1 levels between patients receiving selenium and placebo (70.6 \pm 21.9 vs. 84.6 $\pm 24.7 ; p=0.039)$. These results confirmed that 


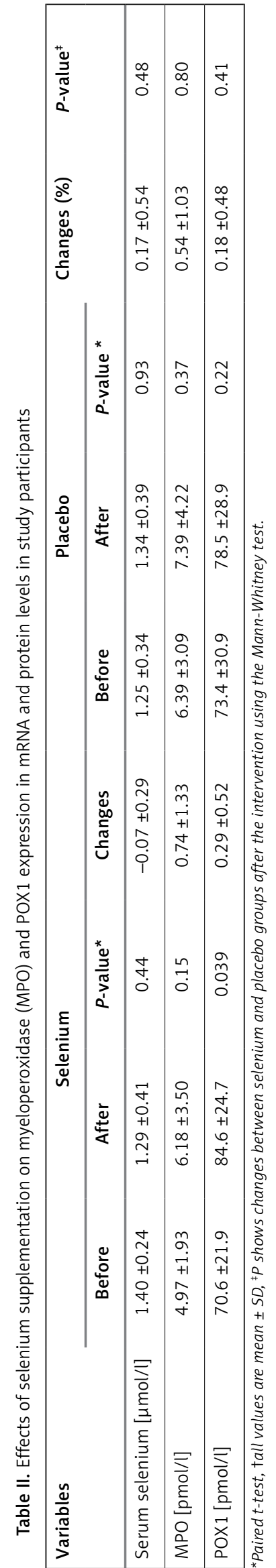

selenium supplementation could increase POX1 activity in CVD patients, i.e. selenium can serve as a potent antiatherogenic factor in atherosclerosis.

It seems that selenium supplementation could affect the level of POX1 levels and increase it, which could be affected by various factors, such as micronutrient dietary intake. Our results of the baseline analysis showed no significant difference between intakes of not only nutritional selenium but also other macro- and micronutrients. In addition, our patients has good compliance, as all subjects who did not use selenium for any reason were excluded from the study. However, in this study we did not assess genetic variation in the genome.

POX1 and PON3 prevent the formation of oxidized-LDL, which is the form of LDL present in foam cells of an atheromatous plaque. Because of their known association with HDL and their effect on oxidized-LDL, POX1 and PON3 are believed to lower the risk of coronary artery disease [18].

The mean levels of MPO and all other parameters did not change significantly after the intervention. MPO may play a role in thermogenesis in humans. Individuals with total or subtotal MPO deficiency, a defect with a frequency of $\approx 1$ in every 2000 to 4000 whites, appear less likely to develop CVDs [19].

Furthermore, individuals harboring a promoter polymorphism associated with a reported twofold reduction in MPO expression appear cardioprotected with markedly reduced angiographic evidence of coronary artery disease, nonfatal myocardial infarction, and cardiac death [9]. Increasing systemic levels of MPO have also been demonstrated to predict the presence of angiographic coronary artery disease [10]. Individuals who possess MPO levels in the fourth quartile among sequential subjects undergoing diagnostic cardiac catheterization at a tertiary referral center were 15-20-fold more likely to demonstrate abnormal coronary angiograms (defined as $>50 \%$ stenosis in one or more major coronary arteries) compared with subjects in the lowest quartile. This relationship remained significant after statistical adjustments for Framingham risk score and C-reactive protein. In addition, plasma and serum levels of MPO have been shown to predict risks of subsequent major adverse cardiac events (nonfatal myocardial infarction, death, and need for revascularization) in patients presenting with either chest pain or acute coronary syndromes [20-23]. Numerous biochemical and genetic studies in humans have demonstrated strong and independent relationships between MPO and CVD risks.

According to previous research, there is a rise in the levels of circulating markers of free radical-induced impairment and a reduction in antioxidant defense levels in CVD [24-26]. Depletion 
of the antioxidative defense system can lead to an increase in the generation of reactive oxygen species (ROS) [27]. The increased oxidative status in CVD patients and subsequent inactivation of POX1, together with low POX1 expression, could serve as major factors responsible for the reduced POX1 activity in CVDs. Considering the involvement of oxidative stress in the development of diabetes and also POX1 activity/inactivation, the administration of antioxidants might protect POX1 inactivation caused by ROS and thus ameliorate diabetes [28-31].

Ghaffari et al. observed that vitamin E and selenium supplementation had hypolipidemic, hypoglycemic, and antioxidative effects in diabetic rats. It could also slow down the progression of diabetic complications through its protective effect on POX1 activity and lipoprotein oxidation [32].

Another study by Strunz et al. indicated that 15 days of Brazil nut consumption significantly increased plasma selenium levels but did not alter serum lipid profile, e.g. HDL, LDL-C, triacylglycerol, apolipoprotein A-I, or apolipoprotein B concentrations, HDL particle diameter, and the activity of antioxidative POX1 [33]. However, there is still controversy over the effectiveness of selenium administration in the prevention of CVD development. Therefore, it seems that despite the published results, further studies are required to elucidate the contribution of oxidative stress to CVD development and the function and role of vitamin antioxidants in cases where ROS and its consequent oxidative damage should be considered in the dietary or nutraceutical management of CVD.

Serum selenium levels are closely related to the living area. Although Iran is located in a low-selenium area of the world [34], we did not find any significant differences in the patients' serum selenium levels after supplementation. Oral supplementation with micronutrients is simple and has been widely employed. In many countries, including the US and France, selenium is considered a supplement rather than a medicine. $100 \mu \mathrm{g}$ daily selenium intake has been recommended by the Brazilian Agency of Sanitary Inspection [35, 36].

If the treatment in this trial turns out to be beneficial, it could be a new and affordable treatment strategy for CVD. After the intervention, the POX1 levels increased slightly, but significantly, in the selenium group compared to the placebo group. So our findings showed that 8 weeks of supplementation with selenium yeast (200 mg selenium/day) caused significant changes in POX1 compared to the placebo group.

The small sample size and the number of participants lost to follow-up constituted the major limitations of this study. In addition, we strongly suggest evaluating the level of MPO and POX1 at the genome level.
In conclusion, our findings suggested that the increased levels of POX1 after selenium supplementation could be attributed to the positive effects of selenium on inhibiting lipid peroxidation (as part of the complicated pathophysiology of CVDs). Therefore, selenium supplementation may play a therapeutic role in CVDs. However, more research with larger sample sizes is required in this field.

\section{Acknowledgments}

The authors appreciate Mrs. Elham Khosravi and Mrs. Narges Jafari for performing laboratory tests. We are also grateful to all patients who participated in this study.

Sources of Financial Support: Isfahan Rehabilitation Research Institute, Isfahan University of Medical Sciences, Isfahan, Iran.

\section{Conflict of interest}

The authors declare no conflict of interest.

\section{References}

1. Sadeghi M, Talaei M, Oveisgharan S, et al. The cumulative incidence of conventional risk factors of cardiovascular disease and their population attributable risk in an Iranian population: The Isfahan Cohort Study. Adv Biomed Res 2014; 3: 242.

2. Talaei M, Sarrafzadegan N, Sadeghi M, et al. Incidence of cardiovascular diseases in an Iranian population: the Isfahan Cohort Study. Arch Iran Med 2013; 16: 138-44.

3. Gharipour M, Sadeghi M, Behmanesh M, Salehi M, Nezafati P, Gharpour A. Selenium homeostasis and clustering of cardiovascular risk factors: a systematic review. Acta Biomed 2017; 88: 263-270.

4. Nazemi L, Nazmara S, Eshraghyan MR. Selenium status in soil, water and essential crops of Iran. Iranian J Environ Health Sci Eng 2012; 9: 11.

5. Motulsky AG. Role of genetic polymorphism of human plasma paraoxonase/arylesterase in hydrolysis of the insecticide metabolites chlorpyrifos oxon and paraoxon. Am J Hum Genet 1988; 43: 230-8.

6. Kunutsor SK, Bakker SJ, James RW, Dullaart RP. Serum paraoxonase-1 activity and risk of incident cardiovascular disease: the PREVEND study and meta-analysis of prospective population studies. Atherosclerosis 2016; 245: 143-54.

7. Kunutsor SK, Kieneker LM, Bakker SJL, James RW, Dullaart RPF. The inverse association of HDL-cholesterol with future risk of hypertension is not modified by its antioxidant constituent, paraoxonase-1: the PREVEND prospective cohort study. Atherosclerosis 2017; 263: 219-26.

8. Schultz J, Kaminker K. Myeloperoxidase of the leucocyte of normal human blood. I. Content and localization. Arch Biochem Biophys 1962; 96: 465-67.

9. Nicholls SJ, Hazen SL. Myeloperoxidase and cardiovascular disease. Arterioscler Thromb Vasc Biol 2005; 25: 1102-11.

10. Simsek O, Kocael A, Kocael P, et al. Inflammatory mediators in the diagnosis and treatment of acute pancreatitis: pentraxin-3, procalcitonin and myeloperoxidase. Arch Med Sci 2018; 14: 288-96. 
11. Heinecke JW. Mechanisms of oxidative damage by myeloperoxidase in atherosclerosis and other inflammatory disorders. J Lab Clin Med 1999; 133: 321-5.

12. Gharipour M, Sadeghi M, Salehi M, et al. Association of expression of selenoprotein $\mathrm{P}$ in mRNA and protein levels with metabolic syndrome in subjects with cardiovascular disease: results of the Selenegene study. J Gene Med 2017; 19. doi: 10.1002/jgm.2945.

13. Souverein OW, de Boer WJ, Geelen A, et al. Uncertainty in intake due to portion size estimation in 24-hour recalls varies between food groups. J Nutr 2011; 141: 1396-401.

14. Zhang R, Brennan ML, Fu X, et al. Association between myeloperoxidase levels and risk of coronary artery disease. JAMA 2001; 286: 2136-42.

15. Gharipour M, Ouguerram K, Nazih EH, et al. Effects of selenium supplementation on expression of SEPP1 in mRNA and protein levels in subjects with and without metabolic syndrome suffering from coronary artery disease: Selenegene study a double-blind randomized controlled trial. J Cell Biochem 2018 Jun 22. doi: 10.1002/ jcb.26844.

16. Ikeda $\mathrm{Y}$, Suehiro T, Inoue $M$, et al. Serum paraoxonase activity and its relationship to diabetic complications in patients with non-insulin-dependent diabetes mellitus. Metabolism 1998; 47: 598-602.

17. Pulli B, Ali M, Forghani R, et al. Measuring myeloperoxidase activity in biological samples. PLoS One 2013; 8: e67976.

18. Mackness MI, Arrol S, Abbott C, Durrington PN. Protection of low-density lipoprotein against oxidative modification by high-density lipoprotein associated paraoxonase. Atherosclerosis 1993; 104: 129-35.

19. Feldman EL. Oxidative stress and diabetic neuropathy: a new understanding of an old problem. J Clin Invest 2003; 111: 431-3.

20. Roman RM, Camargo PV, Borges FK, Rossini AP, Polanczyk CA. Prognostic value of myeloperoxidase in coronary artery disease: comparison of unstable and stable angina patients. Coron Artery Dis 2010; 21: 129-36.

21. Chistiakov DA, Melnichenko AA, Orekhov AN, Bobryshev YV. Paraoxonase and atherosclerosis-related cardiovascular diseases. Biochimie 2017; 132: 19-27.

22. Nicholls SJ, Hazen SL. Myeloperoxidase and cardiovascular disease. Arterioscler Thromb Vasc Biol 2005; 25: 1102-11.

23. Lippman SM, Klein EA, Goodman PJ, et al. Effect of selenium and vitamin $E$ on risk of prostate cancer and other cancers: the selenium and vitamin e cancer prevention trial (select). JAMA 2009; 301: 39-51.

24. Xia Y, Hill KE, Byrne DW, Xu J, Burk RF. Effectiveness of selenium supplements in a low-selenium area of China. Am J Clin Nutr 2005; 81: 829-34.

25. Kaya MG, Yalcin R, Okyay K, et al. Potential role of plasma myeloperoxidase level in predicting long-term outcome of acute myocardial infarction. Tex Heart Inst 2012; 39: 500-6

26. Roman RM, Camargo PV, Borges FK, Rossini AP, Polanczyk CA. Prognostic value of myeloperoxidase in coronary artery disease: comparison of unstable and stable angina patients. Coron Artery Dis 2010; 21: 129-36.

27. Parodi O, De Maria R, Roubina E. Redox state, oxidative stress and endothelial dysfunction in heart failure: the puzzle of nitrate-thiol interaction. J Cardiovasc Med (Hagerstown) 2007; 8: 765-74

28. Rahman K. Studies on free radicals, antioxidants, and co-factors. Clin Interv Aging 2007; 2: 219-36.
29. Yetik-Anacak G, Catravas JD. Nitric oxide and the endothelium: history and impact on cardiovascular disease. Vascul Pharmacol 2006; 45: 268-7.

30. Birben E, Sahiner UM, Sackesen C, Erzurum S, Kalayci O. Oxidative stress and antioxidant defense. World Allergy Organ J 2012; 5: 9-19.

31. Wu Y, Tang L, Chen B. Oxidative stress: implications for the development of diabetic retinopathy and antioxidant therapeutic perspectives. Oxid Med Cell Longev 2014; 2014: 752387.

32. Ghaffari T, Nouri M, Irannejad E, Rashidi MR. Effect of vitamin $E$ and selenium supplement on paraoxonase-1 activity, oxidized low density lipoprotein and antioxidant defense in diabetic rats. Bioimpacts 2011; 1: 121-8.

33. Strunz CC, Oliveira TV, Vinagre JC, Lima A, Cozzolino S, Maranhão RC. Brazil nut ingestion increased plasma selenium but had minimal effects on lipids, apolipoproteins, and high-density lipoprotein function in human subjects. Nutr Res 2008; 28: 151-5.

34. Nazemi L, Nazmara S, Eshraghyan MR. Selenium status in soil, water and essential crops of Iran. Iranian J Environ Health Sci Eng 2012; 9: 11.

35. Kieliszek M, Błażejak S. Current knowledge on the importance of selenium in food for living organisms: a review. Molecules 2016; 21: pii: E609.

36. Kieliszek M, Błażejak S. Selenium: significance, and outlook for supplementation. Nutrition 2013; 29: 713-8. 